\title{
DEUX ARCHERS ASSYRIENS À MARI (SYRIE). ESQUISSE SUR L’INTRODUCTION DU FER AU PROCHE-ORIENT ANCIEN
}

\author{
POR \\ JUAN-LUIS MONTERO FENOLLÓS
}

\begin{abstract}
RESUMEN - ABSTRACT
Este artículo es un estudio sobre dos tumbas del cementerio medio-asirio de Mari (Siria). La presencia de puntas de flecha de hierro entre el ajuar funerario ha servido para realizar una aproximación al proceso de introducción de este nuevo metal en el Próximo Oriente durante el Bronce Reciente. Tanto la documentación escrita como la arqueológica demuestran que el hierro era considerado como un metal precioso y, como tal, usado por las élites asirias para mostrar su posición social.

This paper is a study about two graves from the Middle Assyrian cemetery in Mari (Syria). The presence of iron arrowheads between the funeral furniture has served to make an approach to the process of introduction of this new metal in the Near East in the Late Bronze Age. The written and archaeological documentation demonstrates that the iron was considered as a precious metal, used by Assyrian elites to show its social position.
\end{abstract}

\section{PALABRAS CLAVE - KEY WORDS}

Flechas, Carcaj, Tumbas asirias, Hierro, Mari, Siria.

Arrowheads, Quiver, Assyrian graves, Iron, Mari, Syria.

Depuis soixante-dix ans de recherches sur le tell de Mari, la capitale du Moyen-Euphrate syrien, on sait que trois villes différentes se sont succédées jusqu’à l’incendie provoqué par les troupes du roi Hammurapi de Babylone en 1760 av. J.-C. La cité a survécu à cette destruction un certain temps (période de Khana), mais comme l'ombre d'elle-même. En fait, la ville n'a plus joué aucun rôle important du point de vue politique et économique. Le destin de Mari en tant que capitale royale restera scellé sous les restes de sa splendeur (Margueron, 2000).

Une autre phase d'occupation postérieure à la période urbaine a laissé d'importantes traces dans la stratigraphie du site, bien qu'il soit impossible de préciser pour le moment l'importance de Mari à cette époque. Les premières campagnes de fouilles ont montré qu'il existait une grande nécropole médio-assyrienne dans les ruines du palais amorrite détruit par les babyloniens. De plus quelques vestiges d'un niveau d'habitation à caractère domestique de la même période ont été retrouvés au «chantier E», sur le rebord nord-ouest du tell principal (Margueron et alii, 1993: 17). Nous sommes devant les restes de la petite bourgade médio-assyrienne, qui a donné les tombes retrouvées par André Parrot sur les ruines du grand palais.

Au cours de la troisième campagne (hiver 1935-36), le fouilleur français a mis à jour près de 150 sépultures dotées d'un riche et varié mobilier funéraire (Parrot, 1936: 82). Les grandes cours de l'ancien palais amorrite deviennent des enclos funéraires, où la colonie assy- 
rienne a enterré ses défunts. La plupart des tombes étaient placées et groupées à l’intérieur des cours 106 (cimetière 1) et 131 (cimetière 2). On peut classer les tombes de Mari dites médio-assyriennes en quatre types différents: pleine terre, doubles cloches (deux jarres rapprochées deux à deux, ouverture contre ouverture), jarres et sarcophages (Jean-Marie, 1999: 53). Les deux premiers types sont les plus nombreux.

Le cimetière assyrien de Mari, constitué de près de 400 tombes, n'a jamais été l'objet d'une étude exhaustive et monographique. Elle est encore à faire. Néanmoins, nous disposons depuis quelques années d'un catalogue très utile des tombes trouvées à Mari entre 1933 et 1993 (Jean-Marie, 1999). Mais il nous manque une véritable étude sur le riche mobilier en métal de la nécropole assyrienne, qui est, d'autre part, la plus importante de la région. Dans ce contexte, l'objectif de cet article sera donc la présentation d'une étude détaillée de deux tombes (T.134 et T.176) retrouvées entre février et mars 1936 par André Parrot ${ }^{1}$. Le choix n’a pas été laissé au hasard. Nous avons choisi deux tombes qui contenaient un mobilier en métal très intéressant pour différentes raisons: la présence d'un important lot de pointes de flèches, même d'un carquois; et surtout l'utilisation du fer pour la fabrication d'armes à une époque très ancienne. En fait, nous avons dans les deux tombes de Mari des éléments d'approche à l'art de la guerre dans une période (la fin du IIe millénaire av. J.-C.) marquée par de complexes changements au Proche-Orient. L'effondrement de la civilisation du Bronze Récent laisse la place à l'élaboration d'un nouveau monde construit sur les ruines du XIIe siècle av. J.-C. Le fer appartient à ce nouvel âge.

D’autre part, il faut souligner qu'on connaît bien l'armée de Mari grâce aux célèbres archives de l'époque paléo-babylonienne (Sasson, 1969; Abrahami, 1991; Durand, 1998); on connaît bien aussi l'armement et l'organisation militaire de l'Assyrie au Ier millénaire av. J.-C., fondement de sa politique expansionniste (Malbran-Labat, 1982). Mais, par contre, nous n'avons pas beaucoup de renseignement pour la période médio-assyrienne. L'étude proposée des deux tombes mariotes nous permet de réaliser, aussi succinte soit-elle, une esquisse de la technologie au service de l’armée des assyriens aux derniers siècles du IIe millénaire av. J.-C.

\section{LES TOMBES.}

La tombe T.134 (fig. 1: a) était du type double cloche (deux jarres à base annulaire couchées). Elle était placée sur le mur est de la cour 106 du grand palais (cimetière 1). Le squelette était couché sur le dos, tête à l’est tournée vers le sud, mains à hauteur du crâne et jambes fléchies (Jean-Marie, 1999: 120). Le mobilier funéraire (fig. 2 et 4) était composé de:

\begin{tabular}{|l|l|l|l|}
\hline N $^{\text {PARROT }}$ & N $^{\mathrm{L}}$ LOUVRE & OBJET & MATÉRIAU \\
\hline M.1234 & AO.29721 & Passoire-entonnoir & Cuivre ou bronze \\
\hline M.1235 & AO.19494 & Carquois & Cuivre ou bronze \\
\hline M.1236 & AO.19494 & 5 pointes de flèches & Fer \\
\hline$?$ & $?$ & Bol & Cuivre ou bronze \\
\hline$?$ & $?$ & Bracelet & Cuivre ou bronze \\
\hline
\end{tabular}

1 Le mobilier en métal des deux tombes assyriennes a été étudié pendant un séjour en décembre 1999 à Paris. Je dois remercier Mme. Annie Caubet, Conservateur général chargée du département des Antiquités Orientales du Louvre, pour m’avoir permis de réaliser cette étude; ainsi que Mme. Elisabeth Fontan, Conservateur en chef au même département, pour sa disponibilité durant mon séjour. Je voudrais également remercier le professeur Jean-Claude Margueron, directeur de la Mission de Mari, pour m’avoir confié l'étude des métaux du site. 
La tombe T.176 (fig. 1: b) était aussi du type double cloche et située dans le dallage de la zone sud de la cour 131 (cimetière 2). Le défunt était couché sur le dos, tête à l'est tournée vers le nord, bras croisés sur le thorax et jambes allongées (Jean-Marie, 1999: 124). Le mobilier (fig. 3 et 5) était formé par:

\begin{tabular}{|l|l|l|l|}
\hline No $^{\text {PARROT }}$ & N $^{\text {L LOUVRE }}$ & OBJET & MATÉRIAU \\
\hline M.1301 & Musée d'Alep $\left(\mathrm{n}^{\text {o }}\right.$ ?) & Pyxide & Céramique glaçurée \\
\hline M.1302 & AO.19023 & 23 pointes de flèches & Cuivre ou bronze \\
\hline$?$ & AO.19023 & Bague & Cuivre ou bronze \\
\hline$?$ & $?$ & Anneau & Fer \\
\hline$?$ & $?$ & 2 tiges & Cuivre ou bronze \\
\hline$?$ & $?$ & Coupe & Céramique \\
\hline
\end{tabular}

\section{LA DATATION.}

Depuis sa découverte en 1936, la nécropole installée dans les ruines du grand palais de Zimri-Lim a été qualifiée d'assyrienne et datée vers le milieu du IIe millénaire av. J.-C. (Parrot 1936: 82-84). Mais l'étude des objets les plus significatifs du mobilier funéraire a permis de préciser l'époque d'utilisation du cimetière. La présence de divers objets d'origine égyptien nous a permis d'établir une chronologie relative par synchronisme. Dans la tombe T.119 (cimetière 1) il y avait un scarabée en faïence avec le nom inscrit du pharaon Setnakht, de la XXe dynastie de l'ancien Egypte (Beyer, 1979: 66). La tombe 656 (cimetière 2) contenait une plaquette en faïence, en forme de cartouche dont les deux faces sont incisées de hiéroglyphes au nom d'Aménophis III, pharaon de la XVIIIe dynastie (Mallet, 1975: 26). En outre, Parrot dans son rapport de fouilles de la troisième campagne a écrit que M. Moret a cru retrouver, malgré l'usure, le nom du célèbre Ramsès II dans un scarabée (Parrot 1936: 84). Ce qui est important c'est que ces objets, arrivés jusqu'à Mari dès l'Egypte du Nouvel Empire, sont des indices valables pour établir grosso modo la durée de la nécropole mariote. On pense à une utilisation d’à peu près un siècle et demi, entre 1350 et 1200 av. J.-C.

D'autre part, on peut observer que les objets en faïence polychrome sont très nombreux dans le mobilier funéraire. Parmi ceux-ci, il y a des vases très caractéristiques avec couvercle plat à anses, panse côtelée et décoration glaçurée. Il s’agit de véritables pyxides. La tombe T.176 de Mari (fig. 5) contenait un exemplaire (Jean-Marie, 1999: 124). Ce même type de vase est bien attesté en Mésopotamie et dans la région syro-palestinienne à la deuxième moitié du IIe millénaire av. J.-C. (Moorey, 1994: 178ss.). On l’a retrouvé au cœur de l’Assyrie, dans des tombes d'Aššur datées de la période médio-assyrienne (XIV-XIII siècles av. J.-C.) (Harper et alii, 1995: 108). Dans le sud, il apparaît à Babylone à l'époque kassite (JakobRost et alii, 1992: 107). Dans l'ouest, ce type de céramique est attesté à Minet al-Beida (Schaeffer, 1932) et à Alalakh (Woolley, 1955), toujours dans des phases qui ne dépassent pas le XIIe siècle av. J.-C. Même en Iran, à Chogha Zanbil (akk. Dûr Untaš) les fouilles ont mis à jour des vases en faïence de la fin du XIVe siècle (Ghirshman, 1968).

En conclusion, on peut dater la nécropole de Mari dans la phase historique dite médioassyrienne qui correspond à la phase archéologique du Bronze Récent II (c.1350-1200 av. J.-C.). 


\section{LES ARMES.}

Dans la nécropole médio-assyrienne de Mari on a trouvé des armes dans quelques tombes (11 tombes sur 384), ce qui représente seulement $2.86 \%$ du total. C'est dans les tombes en double cloche qu'il y a le plus d'armes. Parmi celles-ci, aucune épée, ni poignard, ni hache, etc. Les seules armes récupérées furent des pointes de flèches, aussi bien en cuivre/bronze qu'en fer. Les tombes T.134 et T.176 contenaient des exemplaires de ces armes propulsées qui représentent deux traditions métallurgiques tout à fait différentes.

Le mobilier de la tombe T.176 était composé de 23 pointes de flèches en cuivre/bronze (fig. 3). Il s'agit d'un modèle à lame losangique, nervure centrale et longue soie comme système de fixation. La longueur peut varier entre 6.3 et $9.4 \mathrm{~cm}$, mais la plupart ont des dimensions situées autour de $6 \mathrm{~cm}$. La seule exception est une flèchette d'un type très simple de $4 \mathrm{~cm}$ de long. La plupart des exemplaires conservaient des restes de fibres végétales (peut être du roseau) dans la soie. En fait, le roseau était un matériau dur et flexible, facile à travailler et pouvant recevoir une pointe de flèche. Quelques exemplaires ont été trouvés en Egypte en bon état de conservation (Yadin, 1963: 81).

Les pointes de flèches de la tombe T.176 appartiennent à un type très connu dans la phase finale du Bronze Récent de la région syro-palestinienne, où il a été fabriqué en bronze et en cuivre à l'arsenic (Montero Fenollós, 1997: 205 et 210). En Syrie on connaît des exemples datés du XIIIe siècle av. J.-C. à la forteresse de Tell Sabi Abyad (Akkermans et alii, 1993: 47), aux niveaux de la phase Mitanni de Tell Brak (Oates et alii, 1997: 114), à Émar (Beyer, 1982: 119), et au palais nord de Ras Ibn Hani (Bounni et alii, 1998: 69), entre autres sites.

La tombe T.134 contenait 5 pointes de flèches en fer, à lame losangique, barre à section circulaire et soie courte (fig. 2). Elles ont des pointes de grande taille, car leur longueur est d'entre 13 et $8 \mathrm{~cm}^{2}$. Ce petit lot de flèches a été trouvé dans un objet exceptionnel, un carquois en cuivre/bronze. Des pointes de flèches comparables proviennent de la fouille du Fort Salmanazar de Nimrud, en Assyrie (Stronach, 1958: 179). L'analyse microscopique d'un exemplaire a mis en évidence qu'il s'agit d'une arme élaborée à partir d’un fer carburé, c'est-à-dire, d'acier (Curtis et alii, 1979: 378). L'étude d'une pointe de flèche de Toprak Kale (Urartu) montre un fer très carburé et martelé à une température de $600^{\circ} \mathrm{C}$ après carburation (Curtis et alii, 1979: 386). Malheureusement, nous n'avons pas, pour le moment, d'analyses techniques des pointes de flèches en fer de Mari. Dans les textes cunéiformes médio-assyriens on connaît trois types de pointes de flèches en fer: $m u l m u l l u^{3}$, šak $\bar{u} d u(m)^{4}$ et šiltākhu(m) .

Le carquois de métal de Mari (fig. 2 et 4) portait un décor organisé en deux bandes de cercles en relief. Il était composé de deux lames très fines (1 $\mathrm{mm}$ de grosseur) semicirculaires et un fond fixé avec des petits rivets. Il s'agit d'un carquois (išpatu(m) en akkadien $)^{6}$ de petites dimensions, dont la longueur était de $25.5 \mathrm{~cm}$ et $6 \mathrm{~cm}$ de diamètre. c'est un objet exceptionnel de par les matériaux utilisés dans sa fabrication (cuivre/bronze) ainsi que par ses dimensions réduites. On connaît quelques carquois en bronze comparables à Uruk, dans le sud mésopotamien (Vans Ess et Pedde, 1992), à War Kabud, au Luristan (Vanden Berghe, 1967: 57), et à Urartu, en Arménie (Vanden Berghe et De Meyer, 1983: 80), mais datés du Ier millénaire av. J.-C. En général les carquois étaient fabriqués en cuir. Cela expli-

2 Il est très difficile d'établir une division claire entre les pointes de flèches, les lances et les javelines. Etant donnée cette difficulté, Philip (1997: 114) a proposé la classification suivante pour Tell Brak: projectiles de petites dimensions (4 cm de longue), de dimensions moyennes $(5-7 \mathrm{~cm})$ et grandes $(8-10 \mathrm{~cm})$. Les exemplaires en fer de Mari peuvent être classés dans le troisième groupe.

3 CAD, M II, 190, s.v. mulmullu: «arrow».

$4 C A D$, Š II, 228, s.v. šakūdu: «arrow».

$5 C A D$, Š III, 449, s.v. šiltākhu: «arrow».

$6 C A D, \mathrm{I} / \mathrm{J}, 257$, s.v. išpatu: «quiver». 
que l'absence d'exemplaires conservés. Nous devons nous reporter aux représentations artistiques égyptiennes de l'époque (Yadin, 1963: 81), car les assyriennes sont plus tardives (Stillman et Tallis, 1982: 159-174). Néanmoins, dans la documentation cunéiforme du Bronze Récent il y avait des carquois en or, en argent et en bronze (Salonen, 1965: 78). D'après un texte de Nuzi (XVe siècle av. J.-C.) un carquois pouvait contenir jusqu'à 30 pointes de flèches. À Ugarit, chaque archer avait dans son carquois une moyenne de 10 pointes de flèches (Vita, 1995: 151). Le carquois de Mari contenait, nous l'avons vu, 5 flèches en fer.

\section{LE FER.}

Un aspect vraiment intéressant est l'utilisation du fer pour la fabrication des pointes de flèches de la tombe T.134. En fait, la nécropole médio-assyrienne de Mari nous a fourni un important lot d'objets en fer (80 objets) datant d'une période pour laquelle nous n'avons que très peu de renseignements (graphique 1). L'évidence archéologique sur la métallurgie du fer en Mésopotamie avait une distribution géographique et chronologique très limitée. Les principales sources d'objets en fer assyriens sont les trois grandes capitales du Ier millénaire av. J.-C.: Khorsabad, Ninive et Nimrud (Pleiner et Bjorkman, 1974: 284). En Assyrie, il n’y a pas d'objets archéologiques en fer datés avant du VIIIe siècle av. J.-C. (Moorey, 1994: 289). Par contre, Mari a mis à notre disposition une superbe documentation qui nous permet de connaître une phase clé (la fin du Bronze Récent) de l'introduction de la métallurgie du fer au Proche-Orient.

Les archives administratives du palais de Mari (XVIIIe siècle av. J.-C.) ont fourni une documentation exceptionnelle relative au fer, du fait de son ancienneté. Le fer était employé pour élaborer surtout des parures (anneaux, bracelets et bagues) offerts en cadeau, mais aussi des armes (Limet, 1984). À cette époque le fer avait le statut de métal précieux, à en déduire par sa valeur (Bjorkman, 1989: 12).

Sur l'apparition du fer (parzillu( $m$ ) en akkadien) ${ }^{7}$ dans le pays assyrien nous avons quelques renseignement dans les textes à partir du XIIIe siècle av. J.-C. Le roi Salmanazar Ier (1274-1245 av. J.-C.) à propos de la reconstruction du temple Ehursagkurkurra d'Aššur fait introduire dans le dépôt de fondation «stones, silver, gold, iron, copper, tin, etc.» (Grayson, 1972: 84). Dans une tablette administrative contemporaine on mentionne un poignard en fer et peut être une pointe de lance aussi en fer (Postgate, 1973: 13-14). Le roi Ninurta-tukultîAššur (c.1133 av. J.-C.) avait, entre les membres de sa court, un forgeron a son service, du nom de Marduk-bêl-usur (Weidner, 1936: 19). Quelques années plus tard, Tiglath-phalazar Ier (1114-1076 av. J.-C.) nous dit qu'il a chassé des taureaux dans le pays de Mitanni avec son arc et des flèches (type mulmullu et šukūdu(m)) en fer (Grayson, 1976: 16).

En Syrie, nous avons quelques données sur le premier usage du fer dans différentes archives cunéiformes du Bronze Récent. L’inventaire de Qatna (XVe siècle av. J.-C.) a montré l'emploi du fer comme métal précieux (Virolleaud 1928: 92). Une tablette d'Ugarit fait référence à un poignard en fer, peut être envoyé par un dignitaire hittite (Nougayrol, 1970: 6). Dans l'archive d'El-Amarna, où se conserve la correspondance entre le roi Tušratta de Mitanni et les pharaons Aménophis III et Akhénaton, apparaissent des armes et des parures en fer entre les listes de cadeaux royaux (Moran, 1992: 51-57). Les tablettes d'Alalakh contiennent aussi diverses références au fer (Wiseman, 1953: 107).

Les évidences archéologiques sur le fer en Mésopotamie à l'âge du Bronze Récent sont très faibles. Il s'agit d'une demie-douzaine d'objets originaires de Nuzi, Aqar Quf, Aššur et Tell Zubeidi (Moorey, 1994: 288). En Syrie, la collection d'objets en fer datés entre le XVe

\footnotetext{
7 AHw, II, 837, s.v. parzillu(m): «eisen».
} 
et le XIIIe siècle av. J.-C. est très petite aussi: une hache d'Ugarit (Fortin, 1999: 98) des anneaux de Minet al-Beida, et une pointe de flèche, une spatule et des fragments d'Alalakh (Waldbaum, 1978: 17-18). À Hama (périodes I-III, c.1230-875 av. J.-C.), les fouilles danoises on mis à jour un lot de 208 objets en fer (armes, outils et parures). La distribution par phases est la suivante: phase I (1230-1100), 35 objets, phase II (1100-1000), 78 objets, et phase III (1000-875), 95 (Waldbaum, 1978: 27-29). Dans la région nord-orientale de la Syrie, les récentes recherches archéologiques ont mis à jour un anneau en fer à Tell Sabi Abyad (Akkermans et alii, 1993: 30) et une pointe de flèche, ainsi que quelques fragments de fer à Tell Brak datés du Bronze Récent (Oates et alii, 1997: 123).

\section{L'INTERPRÉTATION.}

Après ce parcours à travers des évidences textuelles et archéologiques sur le fer à l'âge du Bronze Récent dans la région syro-mésopotamienne, il est évident que la documentation retrouvée au cimetière médio-assyrien de Mari dévient importante pour l'étude de cette nouvelle technologie. C'est une collection de 80 objets en fer, ce qui représente $19 \%$ du total d’objets en métal du mobilier funéraire de la nécropole (graphique 1). Il faut noter qu’il s'agit d'un pourcentage très similaire à celui des objets en or et en argent (18\%). Ces objets en fer ont été trouvés en un nombre réduit de tombes (24 sur 384). C'est à peu près $6 \%$ du total de tombes fouillées.

Les parures (bagues, anneaux à cheville et bracelets) constituent le groupe le plus important d'objets en fer de Mari (70 \%). Par contre, les armes en fer sont représentées seulement par 24 pointes de flèches (graphique 2) distribuées dans trois tombes: T.134 (5 exemplaires), T.368 (1 exemplaire) et T.506 (18 exemplaires).

Cette information apportée par l'étude du mobilier en métal de la nécropole mariote doit être mise en rapport avec les paroles suivantes: «We must see the early occurrence of iron in Assyria of the late second millennium - it was a metal reserved for kings» (Pleiner et Bjorkman, 1974: 286). D’après les données de Mari, il est évident que pendant la période 1350-1200 av. J.-C. le fer était considéré comme un métal précieux, comparable par sa valeur à l'or et à l'argent. Au début, le fer était un métal cher utilisé par les élites de la société proche-orientale. Étant donnée la rareté et la difficulté pour travailler ce métal, le fer devient un produit chargé de prestige. En Syrie, on ne connaît pas de dépôts de fer exploités à l’âge du Fer (Waldbaum, 1978: 61). Le problème de l'approvisionnement s'est certainement posé rapidement.

Les élites assyriennes ont trouvé dans le fer un moyen de manifester leur position privilégiée. À notre avis, nous sommes devant une situation semblable à celle connue par la région avec l'introduction de l'étain (= bronze) au début du IIIe millénaire av. J.-C. À partir du VIIIe et VIIe siècles av. J.-C. le fer dévient un métal d'usage quotidien, utilisé dans la fabrication de différents types d'outillage et d'armes (Pleiner et Bjorkman, 1974: 296).

Le fer, mais aussi le bronze, vont trouver une grande application dans le célèbre corps d'archers assyriens. La correspondance du temps des Sargonides (c.721-627 av. J.-C.) contient une information très riche sur l'infanterie assyrienne, qui était composée d'éléments légers, archers et frondeurs, et de formations plus lourdes, combattant surtout avec la lance. Les références aux archers sont nombreuses dans la documentation néo-assyrienne (Malbran-Labat, 1982: 78ss.). L'arc (qaštu(m) en akkadien) ${ }^{8}$ et la flèche sont devenus une arme vitale dans les armées du Ier millénaire av. J.-C. à en déduire par leur représentation fréquente dans les scènes de guerres des reliefs des palais néo-assyriens (Curtis et Reade, 1995).

$8 C A D, \mathrm{Q}, 148$, s.v. qaštu: «bow». 
Mais, évidemment, ces représentations en pierre ne permettent pas de connaître le métal employé pour la fabrication des pointes de flèches.

Pendant le Bronze Récent les pointes de flèches pour la bataille étaient en général en bronze ou cuivre arsénié. Par contre, les exemplaires en fer à cette époque sont très rares. La situation va changer de façon radicale à la période néo-assyrienne. La statistique est vraiment convaincante: de 630 pointes de flèches on connaît seulement un exemple en bronze, trouvé à Tell ar-Rimah (Curtis et alii, 1979: 384). Dans ce contexte, les deux tombes médioassyriennes de Mari étudiées sont un véritable paradigme de la situation à la fin de l'âge du Bronze Récent: T.134 (5 pointes en fer) et T.176 (23 pointes en cuivre/bronze). En fait, du total des pointes de flèches récupérées dans la nécropole mariote seulement $28 \%$ sont en fer (graphique 3). En outre, elles sont distribuées dans trois tombes.

Qui sont les individus enterrés dans les deux tombes de Mari ? La réponse à cette question est très difficile, mais d'après l'étude du mobilier funéraire on peut penser que nous sommes devant deux personnes d'une certaine importance sociale. La présence de pointes de flèches et d'un carquois nous fait penser à deux archers liés à l'armée médio-assyrienne. À Ugarit, dans la côte syrienne, les textes administratifs du XIIIe siècle av. J.-C. montrent que l'arc, les pointes de flèches, le carquois, le bouclier, la lance et l'épée courbée sont les armes propres de l'infanterie du royaume. Chaque archer avait dans son carquois une moyenne de 10 pointes de flèches (Vita, 1995: 151-153).

La présence du fer dans des tombes (pour les flèches de la tombe T.134 et un anneau de la tombe T.176) de la période du Bronze Récent est un symbole de prestige. À cette époque, la valeur du fer était très proche à celle des métaux précieux, nous l'avons vu dans les textes. En fait, «In the thirteenth century ... in Assyria ... the use of iron remained more or less limited to the royal court» (Pleiner et Bjorkman, 1974: 307). «Not only in the 13th but in the 12th and beginning of the 11th centuries B.C. iron was still a rare commodity used only for objects intended for royal or religious purposes» (Maxwell-Hyslop, 1974: 140).

L'irruption des assyriens vers le XIVe siècle av. J.-C. dans la région du moyen Euphrate syrien, où se trouve Mari, n'est pas une surprise vue l'existence de Dûr-Katlimmu dans la vallée du Bas Khabur. Dûr-Katlimmu en tant que capitale de province était un centre administratif de contrôle des villes et villages de la région. Elle a joué un rôle clé dans le développement du réseau pour le commerce, surtout des métaux anatoliens (cuivre, étain et fer) ${ }^{9}$ et pour les mouvements des tropes entre les provinces et la capitale de l'empire assyrien (Bonatz et alii, 1998: 110).

Les deux tombes médio-assyriennes de Mari doivent ce comprendre dans ce contexte historique: l'apparition de la technologie du fer et la présence militaire d'Assyrie dans la région. Mais, que représente l'occupation assyrienne de Mari ? Sans doute le blocage de la vallée en utilisant le verrou de Baghuz contre la Babylonie. Il est possible que les individus des tombes T.134 et T.176, sans doute à position privilégiée dans la société de l'époque, soient des officiers de l'armée assyrienne du XIVe ou XIIIe siècle av. J.-C ${ }^{10}$. Peut être ont-ils eu le statut de rab qašti, c'est-à-dire, d'officiers en chef des archers' ${ }^{11}$, liés à la court de DûrKatlimmu.

9 En fait, l'Anatolie est une région très riche en dépôts de fer. Les plus importants sont placés au Taurus et près de la mer Noire (Ryan, 1960: 15-114). Sur l’origine anatolien du fer utilisé par les assyriens cf. l'article de Maxwell-Hyslop (1974).

10 La position isolée des tombes dans la nécropole (fig. 1), mais surtout la présence de pointes de flèches et l'usage du fer sont des arguments solides.

$11 C A D, \mathrm{R}, 31$, s.v. rabû: «official». 


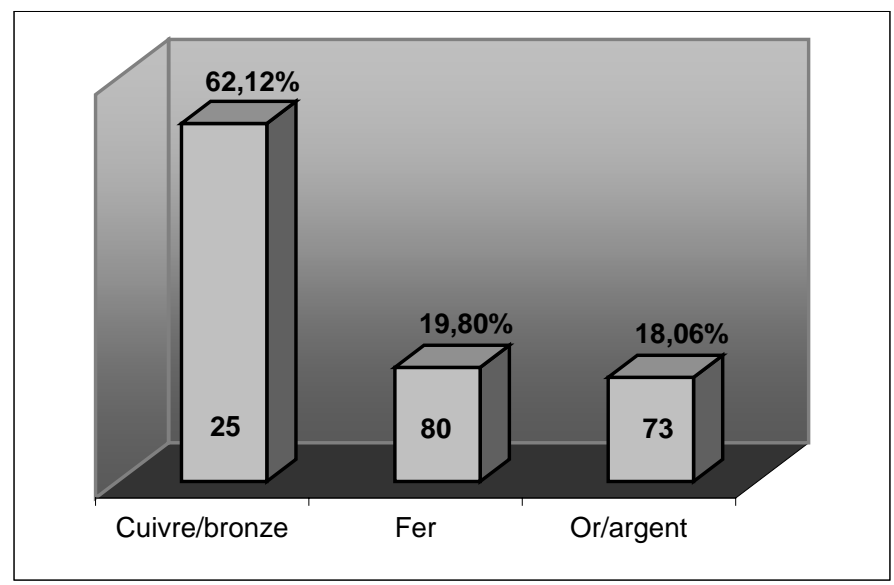

Graphique 1. Pourcentage et nombre d'objets en cuivre/bronze, fer et or/argent dans le cimetière médio-assyrien de Mari.

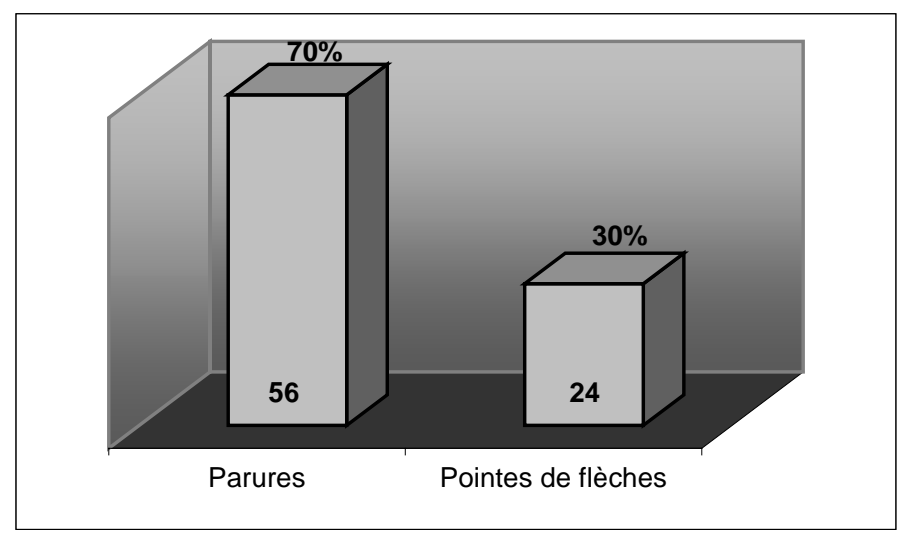

Graphique 2. Pourcentage et nombre d'objets en fer par types dans la nécropole mariote.

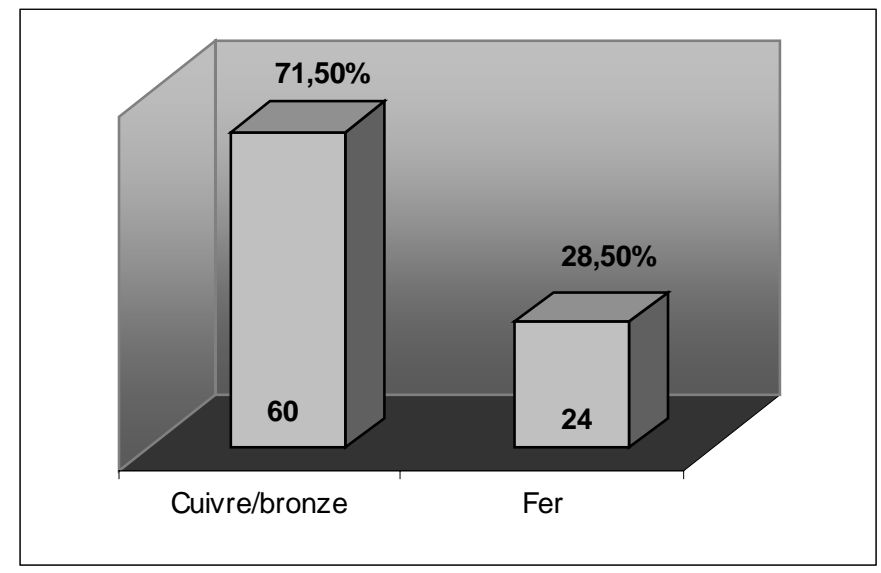

Graphique 3. Pourcentage et nombre de pointes de flèches en cuivre/bronze et fer dans la nécropole de Mari. 


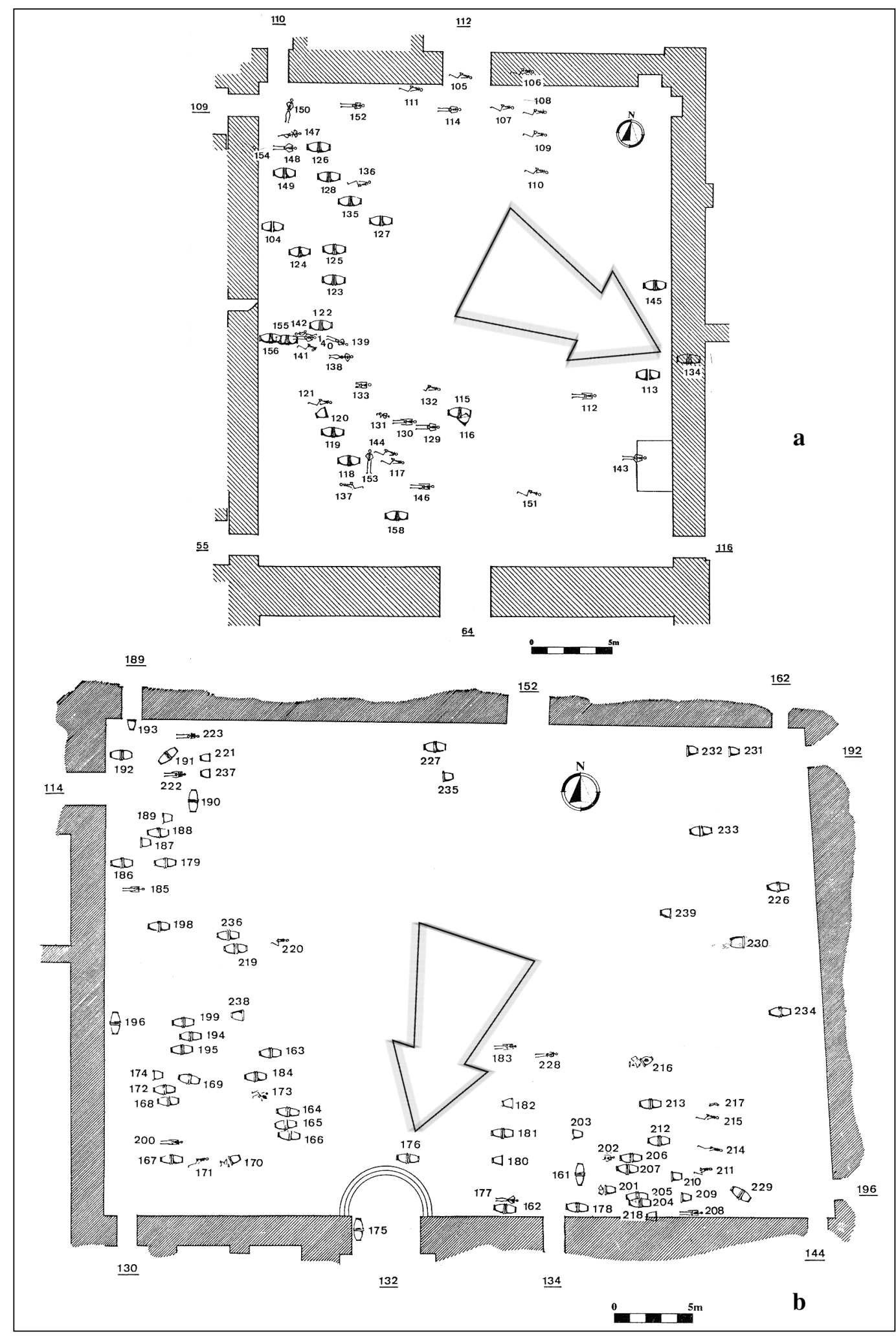

Fig. 1. Situation des tombes T.134 et T.171 dans les cours 106 et 131 du palais de Mari. 


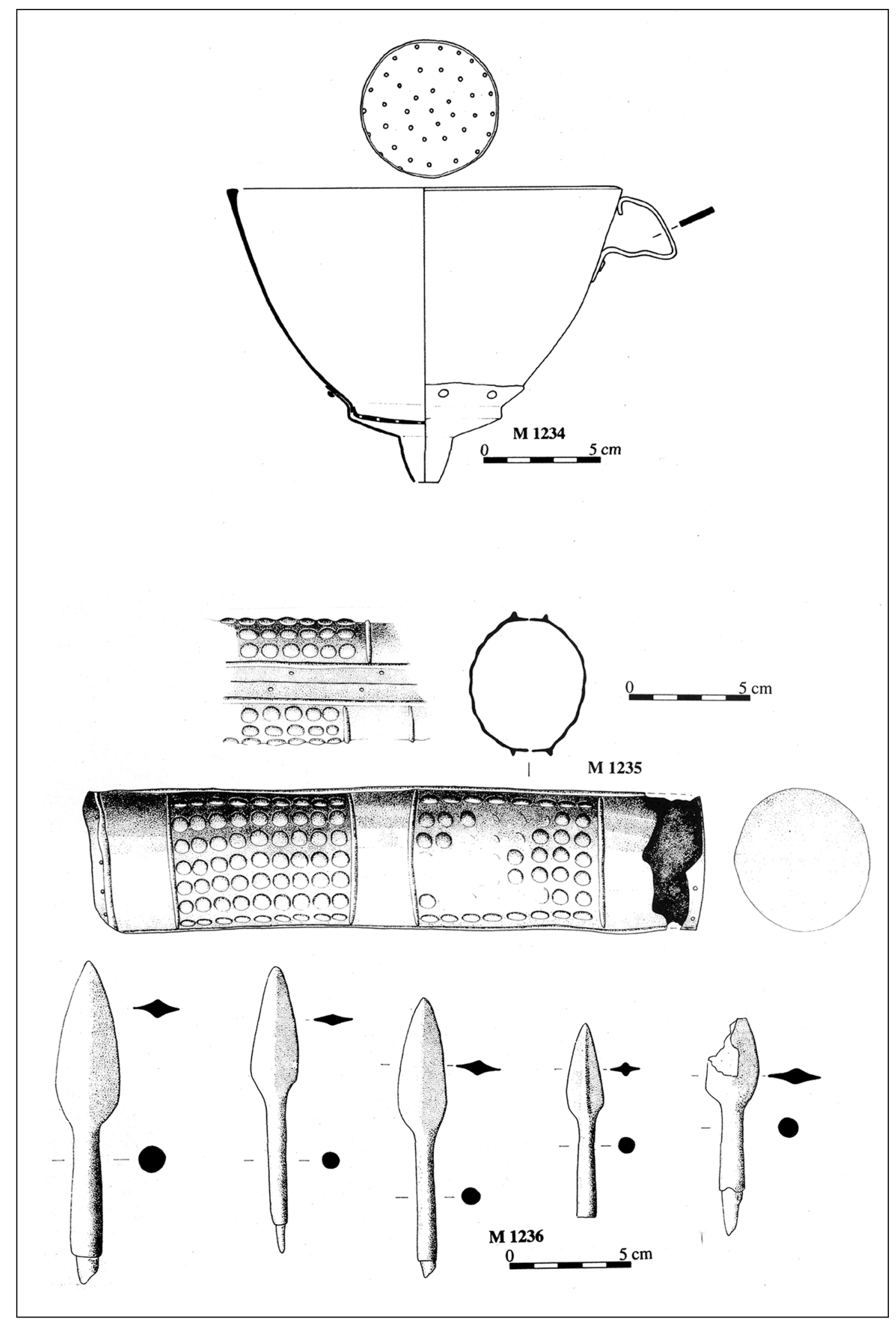

Fig. 2. Mobilier de la tombe T.134 (dessin Musée du Louvre). 


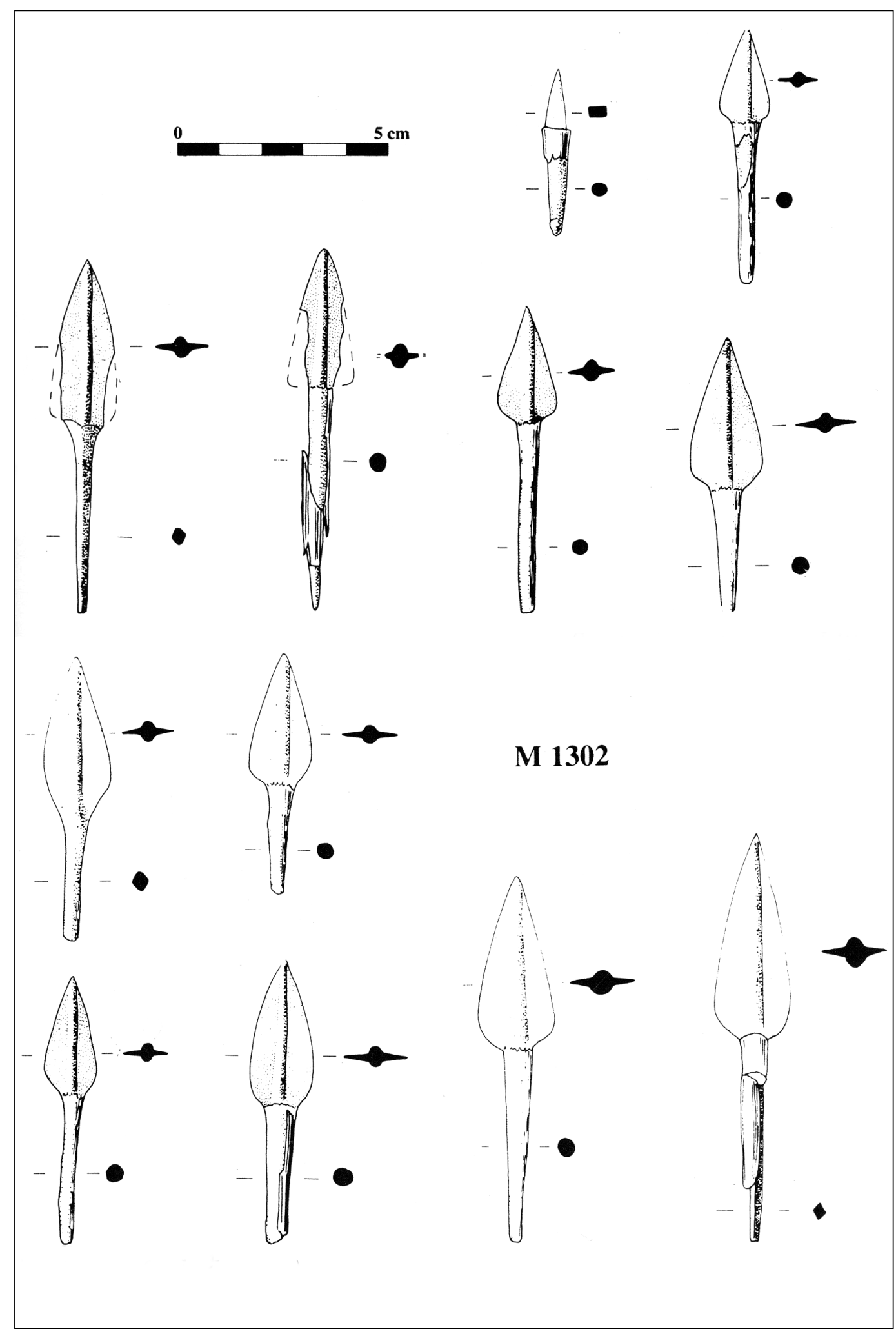

Fig. 3. Mobilier de la tombe T.176 (dessin Musée du Louvre). 


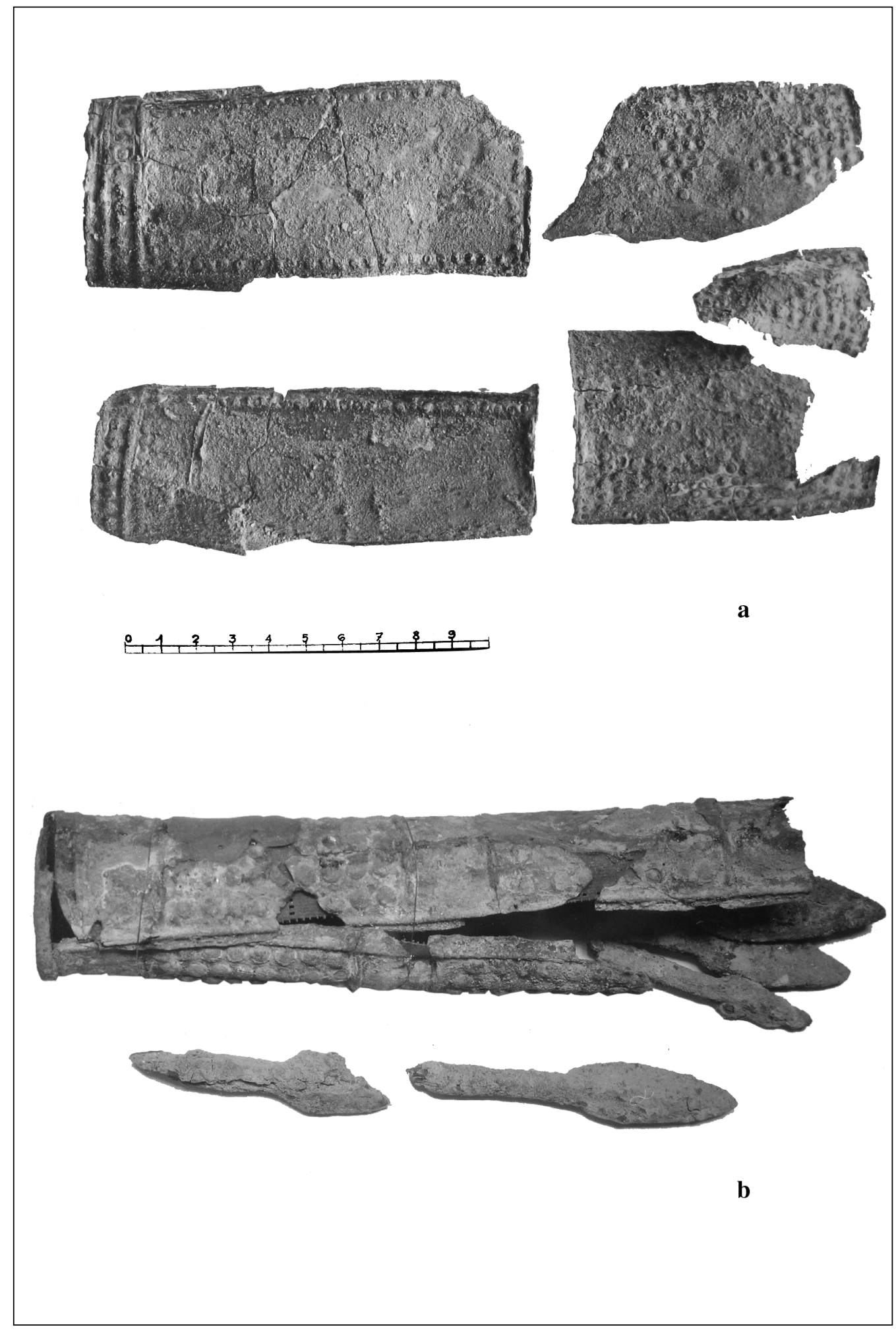

Fig. 4. Carquois en cuivre/bronze et pointes de flèches en fer de la tombe T.134 (photos Archives Mission Archéologique de Mari). 


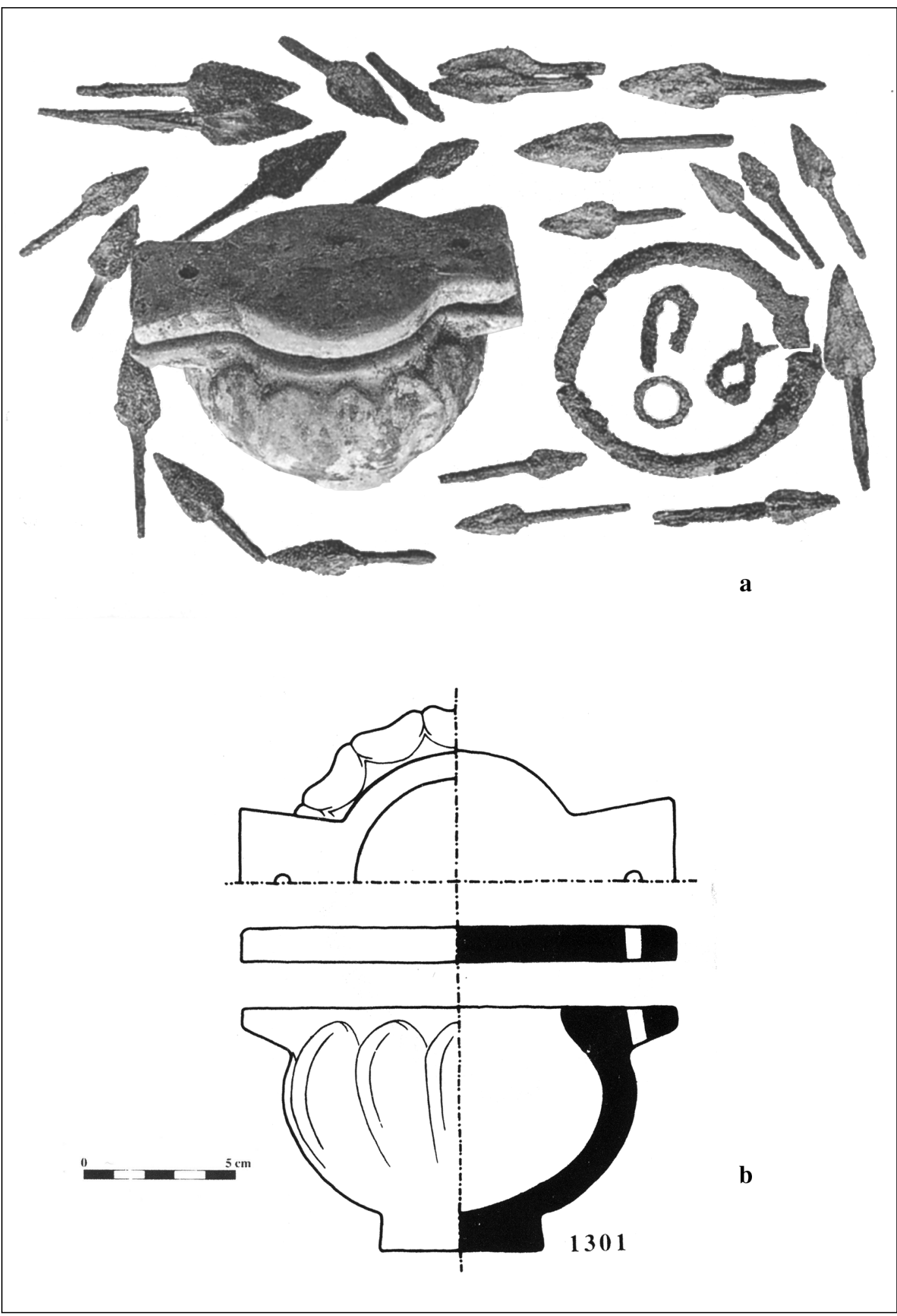

Fig. 5. Pyxide et pointes de flèches de la tombe T.176 (photo Archives Mission Archéologique de Mari). 
JuAN-Luis MONTERo FENOLLÓs

Facultade de Humanidades, Universidade da Coruña, Campus de Esteiro

E-15403Ferrol,fenollos@cdf.udc.es

\section{BIBLIOGRAPHIE}

ABrahami, P. (1991): «L'organisation militaire à Mari». Les Dossiers d'Archéologie, 160: 36-41. AHw = VON SODEN, W. (1972-85): Akkadisches Handwörterbuch. Weisbaden.

AkKermans, P. et alii (1993): «On the Frontier of Assyria: Excavations at Tell Sabi Abyad, 1991». Akkadica, 84-85: 1-47.

BEyeR, D. (1979): De Sumer à Babylone. Collections du Louvre. Paris.

BEYER, D. ed. (1982): Meskéné-Émar. Dix ans de travaux 1972-1982. Paris.

BJoRKMAN, J.K. (1989): «Second Millennium Price of Iron and Copper». NABU 1989/1: 12-13.

Bonatz, D. et alii (1998): Rivers anad Steppes. Cultural Heritage and Environment of the Syrian Jezireh. Damascus.

BounNI, A. et alii (1998): Ras Ibn Hani, I. Le palais nord du Bronze Récent, Beyrouth.

CAD = GELB, I. J. et alii (1956ss): The Assyrian Dictionary of the University of Chicago. Chicago.

CURTIS, J.E. et alii (1979): «Neo-assyrian Ironworking Technology». Proceedings of the American Philosophical Society, 123: 369-390.

CuRTIS, J.E. et READE, J. (1995): Art and Empire. Treasures from Assyria in the British Museum. London.

DuRAND, J.M. (1998): Les documents épistolaires du palais de Mari, II. Paris.

FORTIN, M. (1999): Syrie, terre de civilisation. Québec.

GHIRSHMAN, R. (1968): Tchoga-Zambil II: Temenos, temples, palais, tombes. Paris.

GRAYSON, A.K. (1972): Assyrian Royal Inscriptions, I. Wiesbaden.

GRAYSON, A.K. (1976): Assyrian Royal Inscriptions, II. Wiesbaden.

HARPER, P.O. et alii (1995): Discoveries at Ashur on the Tigris. Assyrian Origins. New York.

JAKOB-Rost, L. et alii (1992): Das Vorderasiatische Museum, Berlin.

JEAN-MARIE, M. (1999): Tombes et nécropoles de Mari. Beyrouth.

LIMET, H. (1984): «Documents relatifs au fer à Mari». MARI, 3: 191-196.

MALBRAN-LABAT, F. (1982): L'armée et l'organisation militaire de l'Assyrie. Genève-Paris.

MALLET, J. (1975): «Mari: une nouvelle coutume funéraire assyrienne». Syria 52: 23-36.

MARGUERON, J.C. et alii (1993): «Mari: rapport préliminaire sur la campagne de 1987». MARI, 7: 5-38.

MARgueron, J.C. (2000): «Mari: derniers développement des recherches conduites sur le tell Hariri». Ith International Congress on the Archaeology of the Ancient Near East, Roma, 909-921.

MAXWELL-Hyslop, K.R. (1974): «Assyrian Sources of Iron». Iraq, 36: 139-154.

Montero Fenollós, J.L. (1997): «Bronces de Palestina en el Museo de Montserrat». Aula Orientali,s 15: 201-222. 
MoORey, P.R.S. (1994): Ancient Mesopotamien Materials and Industries. The Archaeological Evidence. Oxford.

MORAN, W.L. (1992): The Amarna Letters. Baltimore-London.

Nougayrol (1970): Textes en cunéiforme babylonien des archives du Grand Palais et du Palais sud d'Ugarit. Paris.

OATES, D. et alii (1997): Excavations at Tell Brak. Vol. 1: The Mitanni and Old Babylian periods, Camdridge-London.

PARrot, A. (1936): «Les fouilles de Mari. Troisième campagne (hiver 1935-36)». Syria, 18: 54-84.

PHILIP, G. (1997): «The Metal Objects» in: OATES, D. et alii (1997): Excavations at Tell Brak. Vol. 1: The Mitanni and Old Babylian periods, Camdridge-London, 113-124.

Pleiner, R. et BJoRKMAn, J.K. (1974): «The Assyrian Iron Age. The History of Iron in the Assyrian Civilization». Proceedings of the American Philosophical Society, 118: 283-313.

Postgate, J.N. (1973): «Assyrian Texts and Fragments». Iraq, 35: 9-36.

Reuther, O. (1926): Die Innenstadt von Babylon (Merkes). Leipzig.

RyAn, C.W. (1960): A Guide to the Known Minerals of Turkey. Ankara.

SALONEN, E. (1965): Die Waffen der alten Mesopotamier, Helsinki.

SASSON, J.M. (1969): The Military Establishments at Mari. Roma.

SCHAEFFER, C.F.A. (1932): «Les fouilles de Minet-el-Beida et de Ras Shamra, troisième campagne (printemps 1931)». Syria 13: 1-27.

Stillman, N. et Tallis, N. (1984): Armies of the Ancien Near East. Worthing, Sussex.

StRONACH, D. (1958): «Metal Objects from the 1957 Excavations at Nimrud». Iraq 20: 169-181.

VAN Ess, M. et PEDDE, F. (1992): Uruk: Kleinfunde II, DAI Bagdad.

VANDen Berghe, L. (1967): «La nécropole de War Kabud ou le déclin d'une civilisation du Bronze». Archeologia, 18: 49-61.

VANDEn Berghe, L. et De MeYer, L. (1983): Urartu, Gent.

VirolleAud, C. (1928): «Les tablettes cunéiformes de Mishrifé-Katna». Syria, 9: 80-96.

VITA, J.P. (1995): El ejército de Ugarit. Madrid.

WaldBaum, J.C. (1978): From Bronze to Iron. The Transition from the Bronze Age to the Iron Age in the Eastern Mediterranean. Göteborg.

WEIDNER, E.F. (1936): «Aus den Tagen eines assyriscehn Schattenkönigs». Archiv für Orientforschung 10: 1-52.

Wiseman, (1953): The Alalakh Tablets. London.

WOOLLEY, C.L. (1955): Alalakh. London.

YAdiN, Y. (1963): The Art of Warfare in Biblical Lands. New York. 